\title{
Stars and Cultural Astronomy
}

\author{
Bernadette Brady \\ University of Wales Trinity Saint David \\ b.brady@uwtsd.ac.uk
}

Stars are ubiquitous; the Sun and Moon are singletons. The Sun and the Moon visually dominate the sky. They change in the amount of light they produce, either monthly or yearly. They also rhythmically change how they embrace the horizon, creating a measure of time and seasons. In contrast, the stars are many and their light is small. Their place in the sky is fixed and, ignoring precession, their relationship to the horizon is constant, always rising or setting at the same point. They are different in almost every way to the luminaries, in their multiplicity, light, fixed spatial relationship to each other and fixity in a landscape. These distinct characteristics mean that the stars are a catalyst for sky narratives quite different from those of the luminaries.

The most easily recognisable stellar sky narratives are the constellation stories. The multiplicity of the stars produces a scattering of lights across the night sky according to a fixed pattern which, to the human mind - with its apophenia, the tendency to see patterns - results in the heavens becoming a vast storyboard of constellations and clusters. These stories are placed on bright stars, dim stars, coloured stars, dark gaps or voids, and milky hazes. Every spot of the dome of the heavens holds some culture's myth, some culture's cosmic narrative, and every visible place in the dome of the heavens has been claimed by most cultures. Their consistency of movement and fixedness in orientation to each other, however, also offer humanity something quite unique: a view of eternity.

\section{The Importance of Eternity}

For early hunter-gatherers and agriculturalists, the world was a place of uncertainty in which the threat of sudden hardship or even death was ever-present. All known things were subject to change; however, the night revealed another place: a place of non-change which slowly orbited around a fixed central point, constant in its spatial relationships and with the horizon. This place provided evidence of eternity, a world which could surpasses death and defy time. The author of Epinomis, formerly attributed to Plato (c.428-c.348 BC) has an "Athenian Visitor" who spoke of heavenly creatures as "indestructible, immortal and divine". The Athenian contrasted creatures of two sorts, 
"one made entirely of fire the other of earth, and the earthly kind moves in disorder, while the one of fire moves in perfect order" (Plato [attrib.], Epinomis 982a). There were, therefore, two worlds; one of change made of earth and one of divine fiery eternity revealed in the stars every night.

Long before this, however, the earliest known religious texts, the Pyramid Texts from Egypt's Old Kingdom (2686 BC-2134 BC), also spoke of the eternal and divine stars. The Egyptians of the third millennium $B C$ wrote of the circumpolar stars, those that never set for a given location, and called them the "Imperishable Stars", those that do not know destruction (Allen 2005, 9). The night sky with the eternal circumpolar stars provided the destination of the pharaonic soul, a soul different from all other human souls in its cosmic and divine nature. Upon the death of the king, the pharaonic soul ascended to the Imperishable Stars to take up its role as cosmic ruler. However, it would also live again in the new king's mortal being. All other mortal souls were also linked to the stars; not to the Imperishable Stars, but to those defined as the Unwearying Stars, those that set with the Sun, disappeared from view for weeks or months, and then finally returned as heliacal rising stars. These were called the Unwearying Stars as they never grew tired of the unending cycle of life, death and rebirth. It is the stars, therefore, that provide the visual catalyst for the earliest known writings on the notions of soul, immortality and reincarnation. From this Egyptian example set in the Early to Middle Bronze Age, and its legacy as expressed in Epinomis, one can argue for the generalisation across cultures of a view of the stars providing a glimpse into the eternal world of the divine. Indeed, the stars themselves help to shape our notion of divine.

In the myth of the ascent of the pharaonic soul to the Imperishable Stars, the Egyptians added a heavenly ladder or pathway which enabled the king's soul to reach the sacred centre point of the stars. There are many ways that this can be explored but, by the time of Cicero (106-43 BC) two millennia later, it was the Milky Way that was linked to the ascent of the soul. In the Dream of Scipio, the sixth book of his On the Republic (De Re Publica), he imagines the Roman general Scipio Aemilianus having a mystical encounter with his adoptive grandfather Scipio Africanus the Elder, who shows him the heavens. The vision explains about living a good life in order to achieve a divine one after death:

"[...] Such a life is the road to the skies, to that gathering of those who have completed their earthly lives and been relieved of the body, and who live in yonder place which you now see" (it was the circle of light which blazed most brightly among the other fires), "and which you on earth, borrowing a Greek term, call the Milk Circle."

(On the Republic $6.16=$ Cicero 1928, 269)

In the time of Cicero, the Milky Way was an enigma, as one could see stars within the Milky Way which suggested that the "milk" was of another form of matter and possibly made up of a unique material that linked mortal with the divine, earth with sky. Thus, Cicero's view that it was the final resting place of the soul was feasible.

The Milky Way is today understood as the galaxy to which our solar system belongs; we view it from within and thus see it as a band of stars in our nightly sky. Like all other objects in the heavens, it orbits the celestial pole and thus produces the experience of watching a milky band of light acting like a hoop spinning on one's arm (the pole): some- 
times it will be above the arm, sometimes to the side and sometimes underneath. Hence this milky circle produces a variety of different relationships to any landscape, for the Milky Way is not a homogeneous band of stars. Indeed, it is better to think of the Milky Way as a series of long narrow "constellations" with different visual appearances, all of which engage with a landscape in a consistent manner. In the first century AD, Claudius Ptolemy recorded his observations of the Milky Way in his Almagest ( $8.2=$ Ptolemy 1998, $400-404)$, providing a rare insight into its level of visibility at that time. His description spoke of brightness, varying levels of milky-ness, and dark rifts or voids, providing the researcher today with visuals of the Milky Way some two thousand years ago.

With the varying appearance of the Milky Way there will be times when the horizon is flooded as it lies upon the ground; at other times, it will stand upright on the horizon, providing the phenomenon of a road to the stars. Significantly, for a given latitude, the stars rising or setting that are associated with these different Milky Way positions, in relation to the horizon, will be consistent and not influenced by precession. For example, at the latitude of Cairo the setting of Sirius will always be linked to the Milky Way flooding the horizon, while the setting of Antares will occur as the Milky Way stands upright on the horizon. Precession will influence the times of the year this occurs but not the star and the associated Milky Way visual phenomenon. Furthermore, the Milky Way's range of rising or setting azimuths will embrace a wide arc along the horizon, as it will cover the rising or setting points of a declination range of $130^{\circ}$. Being aware of this range and its resulting azimuth arc is the first step in exploring any possible Milky Way sky narrative for a site.

The phenomenon of the Milky Way is as consistent and stable as the view of the unchanging stars, with both offering a view of permanence in a world otherwise of change. This stellar permanency was, by the Egyptians, interpreted as eternity, a view that can be generalised across other cultures. The builders of megalithic tombs, or of any structure, may have also wished to engage in this unchanging star narrative and to link their dead to the eternal place of the stars. Consequently, for a researcher to avoid asking star questions about a location could well be to ignore the major intention of a site.

\section{The Problem of Precession}

One of the problems with working with stars is that of precession and the uncertainty of the age of a site. However, although the rate of precession is constant, at approximately 50 seconds of arc per year, it is an error to think that this can be applied universally across all stars. For example, some stars are remarkably stable in their declination, such as Dubhe, in Ursa Major. In $2500 \mathrm{BC}$, Dubhe had a declination of $+71^{\circ}$; by $1000 \mathrm{BC}$, this had shifted to $+73^{\circ}$; however, by the first century $A D$, its declination was back to $+71^{\circ}$. Thus, over 2500 years, Dubhe maintained a reasonably stable declination. Closer to the equator, the star Spica, in Virgo, in $4500 \mathrm{BC}$ held a declination of $+20^{\circ}$ and, over the next thousand years, only shifted to $+17^{\circ}$. Later, from $2000 \mathrm{BC}$ to $1000 \mathrm{BC}$, it shifted from $+11^{\circ}$ to $+5^{\circ}$, a movement of $6^{\circ}$, while the declination of Sirius, the brightest star in the sky, moved only from $-18^{\circ}$ to $-16^{\circ}$ between $1500 \mathrm{BC}$ and $1500 \mathrm{AD}$, a period of three thousand years. Hence, for any location, the range of declinations for a given period 
needs to be calculated for each star. Some of these stars will have large shifts in azimuths while others may have almost no changes to their rising and setting locations. Thus given the range of dating for a possible site the rate of shift of azimuth for a particular star may be quite insignificant.

\section{Navigation, Star-Lines and Stones}

The consistency of the stars to each other also provides a reliable navigational tool. In the fourth century BC, Aratus described the constellations in his poem the Phaemonena. In this work, he located the Tropics of Capricorn and Cancer, as well as the equator, by drawing lines through the stars. The actual lines he drew showed that he was not working with direct observation, but rather in some cases was writing down a far older oral tradition. The logic behind these lines being held in the oral tradition was that knowledge of such star-lines (the equator and the tropics) meant that one could have a mental star compass. Thus, in any landscape, these key points on the horizon could be quickly located by finding the current part of the star-line visible in the night sky and projecting it down to the horizon. Hence such star-lines would have been valuable to memorise as well as pass on to later generations.

A community, however, may have had other orientations they wished to preserve. These could be pathways to other tribal lands or communities, all of which could be indicated by a star-line. These important tribal star-lines could be learnt by spending a few nights amongst the communities' stones. In this instance, the influence of precession is nullified, as each new generation would be learning their star-lines from their stones, in much the same way as the Polynesian and Micronesian cultures used stone alignments to teach star-lines for navigation across the pacific (Brady 2015, 60). Thus, a community could maintain its navigational knowledge to important ritual places, burial grounds and clan meetings, locations all designated by the azimuth indicated by different stones or markers. Similarly, it may be that some court cairns or passage tombs, instead of being orientated to a horizon calendar event, could instead be orientated to the star-line pointing to their community.

Finally, the eternity of the stars also offered a consistent measure of time. For example, the bewitching vagaries of the lunar calendar could only be fine-tuned by either a clear indication of the day of the equinox (the solstice would be too slow moving to be of use in pinpointing a single day) or by using the stars. Solar alignments at sites such as Cain T at Loughcrew in Ireland with its equinox illumination may have been used to fine-tune a lunar calendar, while the Egyptians turned to the stars and added a leap lunar month to their lunar calendar if the heliacal rising of Sirius took place in the last eleven days of the lunar month (Parker 1974, 52). In this instant the stars are a more reliable tool than the equinox illumination, as the equinox viewing would be more weather sensitive.

In 1973, Carl Sagan proclaimed, "We are made of star-stuff", linking the material world of elements and solar power to the human body (Sagan and Agel 2000 [1973]: 190). And so too are cultures but, in this case, the star-stuff is not the atoms of suns that make up our flesh but, instead, it is the evidence for the shaping of our souls and the guardians of our time. Available every night, the stars reveal a place that never changes: 
eternity, and this very eternity also offers reliability in navigation and calendar. Stars may be ubiquitous, but to our distant ancestors they were never insignificant.

\section{References}

Allen, J. P., 2005. The Ancient Egyptian Pyramid Texts. Atlanta, Florida: Society of Biblical Literature.

Aratus., 2010. Phaenomena. Baltimore, Maryland: John Hopkins University Press.

Brady, B., 2015. "Star-Paths, Stones and Horizon Astronomy". in SEAC2011 Stars and Stones: Vogages in Archaeoastronomy and Cultural Astronomy, edited by F. Pimenta, N. Ribeiro, F. Silva, N. Campion, A. Joaquinito and L. Tirapicos. British Archaeological Reports, International Series 2720: 58-63. Oxford: Archaeopress.

Cicero, 1928. On the Republic, trans. C. W. Keyes. In Cicero, vol. 16: On the Republic, On the Laws, 1-286. Loeb Classical Library 213. Cambridge, Massachusetts: Harvard University Press.

Parker, R. A., 1974. "Ancient Egyptian Astronomy". In Philosophical Transactions of the Royal Society of London: Series A, Mathematical and Physical Sciences 276 (1257): 51-65. https://doi.org/10.1098/rsta.1974.0009

Plato [attrib.], 1997. Epinomis, trans. R. D. McKirahan. In Plato: Complete Works, ed. J. M. Cooper, 1617-1633. Cambridge: Hackett.

Ptolemy, Claudius, 1998. Ptolemy's Almagest, trans. G. J. Toomer. Princeton, New Jersey: Princeton University Press.

Sagan, C. and J. Agel, 2000 [1973]. The Cosmic Connection: An Extraterrestrial Perspective. Cambridge: Cambridge University Press. 